\title{
The Optimal Allocation of Ocean Space for the Purposes of Fishery and Ecotourism Management
}

\author{
Soovoojeet Jana*, T. K. Kar \\ Department of Mathematics, Bengal En gin eering and Science University, Shibpur, Howrah, 711103, West Bengal, India
}

\begin{abstract}
This paper deals with the optimal allocation of an ocean space for the purposes of fishing and ecotourism manage ment. Here we have considered that one portion of the region to be used for ecotourism and rest of the portion to be used for fishery management. Our main aim of the problem is to optimize the total profit earned from both fishery and ecotourism and for this purpose we have used optimal control technique. Some necessary and sufficient conditions are established for the existence of different equilib ria, their stability and Hopf bifurcations of this optimal control problem. It is observed that the result depend strongly on different catch rate functions of the fishery.
\end{abstract}

Keywords Ecotouris m, Harvesting, Adjustment Cost, Optimal Control, Stability

\section{Introduction}

The excessive and unsustainable exploitation of our marine resources has led to the promotion of marine reserves as a fishery management tool. Marine protected areas (MPAs) where fishing is restricted or prohibited, can offer for the recovery of exploited stock and fishery enhancement. The objectives pursued can generally be classified under one of the three following catagories: ecosystem preservation, the management of commercial fisheries and (or) development of rec reational activit ies. But to the authors' knowledge there is no such work on ecotourism management in the marine reserved portion. But we do think that it is quiet more beneficial to the society if we create ecotourism in the reserved portion of the ocean with the fishery management in the other portion.

Conrad (1999) showed that, in the absence of ecological uncertainity and in the context of optimal harvesting, reserves generate no economic benefits to fishermen. Such result coincides with the perspective of many fishermen and also some economists. However Luke et al. (1998) asserted that MPAs can be viewed as a kind of insurance against scientific uncertainity, stock ass es sments or regulation errors. Kar and Matsuda (2008) consider a bioeconomic model of a single species fishery with a marine reserve. Their study examines the impact of the creation of the marine protested areas (MPAs), from both economic and biological perspectives. In particular, they exa mined the effects of

\footnotetext{
* Corresponding author:

soovoojeet@gmail.com (Soovoojeet Jana)

Published online at http://journal.sapub.org/ms

Copyright (C) 2012 Scientific \& Academic Publishing. All Rights Reserved
}

protected patches and harvesting on resource populations. They conclude that the protected patches are an effective means of conserving resource populations, even though extinction can not be prevented in all cases. Hartmann et al. (2007) investigatede the economic optimality of implementing an MPA to get more informative data about fish population, thereby arrising a better management strategy. Powell et al. (2002), examined the contribution of fully protected tropical marine reserves to fishery enhancement by modelling marine reserves fishery linkages. The consequences of reserve establishment on the long run equilibrium fish biomass and fishery catch levels are evaluated. They also concluded that marine reserves are an important component of sustainable tropical fisheries management and reserves will be most effective when coupled with fishing effort controls in adjacent fisheries.[1] In their books Clark (1990) and Kot (2001) present mathematical study of population ecology with harvesting. Recently Kar and Chakraborty (2009) describes marine reserves and its consequences as a fisheries management tool. But to the authours knowledge no attempt has been made to consider the ecotourism management in the reserved portion of the fishery. [2]

In our present work we divide the total region i.e. total available ocean spaces into two regions: one is the fishing zone and the other one is no fishing zone. The no fishing zone should be used for the ecotourism purpose by arranging boating, some water sports etc. Also it is possible to make the region for ecotouris $m$ purpose in another way. In a comparative higher region of a sea or big lake can be made man-made island where eco-touris $m$ should be taken place by means of tourism, gardening, aquarium etc. It is very useful that the investment cost for the creation of ecotourism is quiet high though thereafter the cost will be less than that of the fishery. 


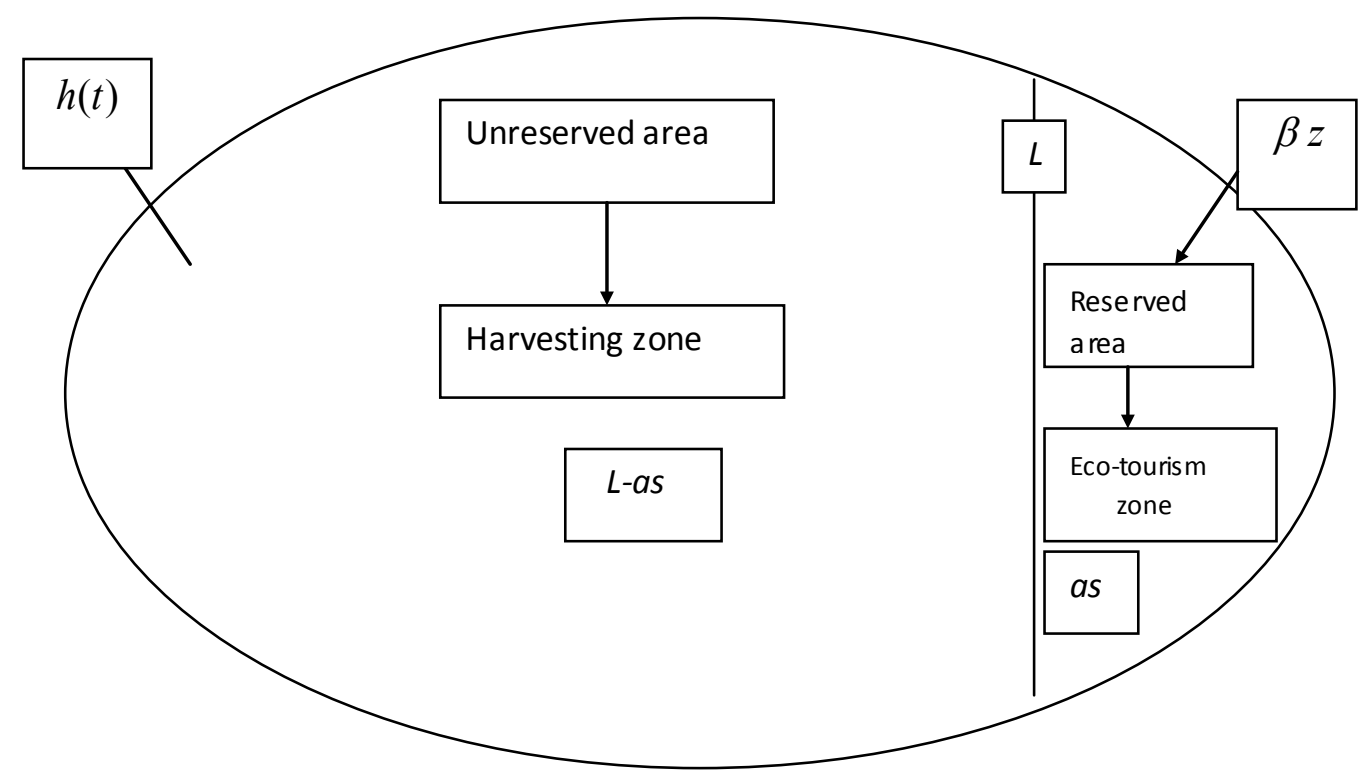

Figure 1. Conseptual diagram of an ocean region allocated for harvesting zone and ecotourism zone

For modelling purpose let us assume that $L$ be the total ocean area where both fishing and eco-tourism are done and $s$ be the portion where only ecotourism takes place. Thus obviously the total region for eco-tourism would be proportional to $S$ and hence it is taken as $a s$ with $a$ is a positive parameter. Also let $r$ be the intrinsic growth rate of the population species in the fishery system and $h(t)$ be their catching rate. Obviously the carrying capacity for this population would be $L$-as. Hence our mathematical model of the single species population is:

$$
\frac{d x}{d t}=r x\left(1-\frac{x}{L-a s}\right)-h
$$

Let $z$ be the total amount of investment applied for eco-tourism purpose. Now if $\beta$ be the proportion parameter i.e. if the portion $\beta(\in[0,1])$ of the total investment cost used for preparing the non fishing zone for eco-tourism zone. The next amount of investment i.e. $(1-\beta) z$ is applied to make some kind of publicity among the tourists so that they are attracted to visit that place. Now we reconstruct our mathematical model as follows:

$$
\begin{aligned}
& \frac{d x}{d t}=r x\left(1-\frac{x}{L-a s}\right)-h \\
& \frac{d s}{d t}=\beta z
\end{aligned}
$$

with the conditions $s(0)=s_{0}, z \in[-a, a], 0<a<\infty$.

Obviously the secondary cost i.e. $(1-\beta) z$ will be proportional to the income due to the eco-tourism. Moreover the income due to the eco-tourism will be prportional to the area where eco-tourism is done. Thus we may take the total income due to the eco-tourism as $p_{2}(1-\beta) s z$. Next we calculate the total amount of profit due to the fishery system. Let us assume that $E$ be the harvesting effort, $c$ be the cost for each unit of harvesting and $p_{1}$ be the selling price of each unit of fish due to the harvesting. Thus total economic rent or profit due to the fishery management is: $p_{1} h-c E$. In figure 1 , we present a conceptual diagram of the considered system.

Moreover there is a cost which is known as "adjustment cost" (see Stollery (1986), Feichtinger and Sorger (1986), Vilchez et al (2004) etc.) is taken as the quadratic function of the variable $z$. This adjustment cost can be interpreted as the cost initiating with the cost due to the eco-tourism. This cost is taken as quadratic function of the variable $z$ since over investment for the eco-tourism may be the cause for the loss in both eco-tourism and harvesting. Now we form the optimal control problem with the fo llo wing Jacobian

$$
J=\max _{z} \int_{0}^{\infty} e^{-\delta t}\left(p_{1} h+p_{2}(1-\beta) s z-c E-\alpha z^{2}\right),(1.3)
$$

subject to the differential equations (1.2).

Here $\delta$ is the discount rate associated with the infinite horizon optimal control problem. Clearly (1.3) is an optimal control problem with two state variables namely $x$ and $s$ and one control variable namely $z$. We use Pontryagain's maximum principle to solve the above optimal control problem. At first we form $H$, the current value Hamiltonian of our problem as follows:

$$
\begin{aligned}
& H=\left(p_{1} h+p_{2}(1-\beta) s z-c E-\alpha z^{2}\right) \\
& +\mu_{1}\left(r x\left(1-\frac{x}{L-a s}\right)-h\right)+\mu_{2} \beta z
\end{aligned}
$$

where $\mu_{1}$ and $\mu_{2}$ are co-state variables.

Now from the optimality condition we have, $\frac{\partial H}{\partial z}=0$ which gives, $p_{2} s-2 \alpha z+\mu_{2}=0$ i.e.

$$
\mu_{2}=\frac{1}{\beta}\left[2 \alpha z-p_{2}(1-\beta) s\right] .
$$


Also we get the transversality conditions of the system as follows:

$$
\begin{aligned}
& \frac{d \mu_{1}}{d t}=\delta \mu_{1}-\frac{\partial H}{\partial x} \\
& \frac{d \mu_{2}}{d t}=\delta \mu_{2}-\frac{\partial H}{\partial s} .
\end{aligned}
$$

Now together with the state equations and the transversality equations we can write our system as a combination of four first order differential equations from which we can get the required solution of our system. Now these equations are as follows:

$$
\begin{aligned}
& \frac{d x}{d t}=r x\left(1-\frac{x}{L-a s}\right)-h, \\
& \frac{d s}{d t}=\beta z, \\
& \frac{d \mu_{1}}{d t}=\delta \mu_{1}-\frac{\partial H}{\partial x}, \\
& \frac{d \mu_{2}}{d t}=\delta \mu_{2}-\frac{\partial H}{\partial s} .
\end{aligned}
$$

Our paper is organized in the following way. The sections 2, 3 and 4 deal with the analysis of the system for three different harvesting functions namely, Schaefer catching function, Cobb-Douglous catching function and Michaelis-Menten type of harvesting respectively. In section 5 , we give some numerical simulations and in the last section we draw some conclusions from our throughout analys is.

\section{Analysis for $h(t)=q E x(t)$}

Schaefer (1957) introduce the harvesting form as catch-per-unit effort. For this here we take harvesting in the form $h(t)=q E x(t)$ where $q$ is the catchibility coefficient. Using the optimality condition, we have from (1.6) the reduced four first order differential equations as follows:

$$
\begin{aligned}
& \frac{d x}{d t}=r x\left(1-\frac{x}{L-a s}\right)-q E x, \\
& \frac{d s}{d t}=\beta z, \\
& \frac{d \mu_{1}}{d t}=\mu_{1}\left(\delta-r+q E+\frac{2 r x}{L-a s}\right)-p_{1} q E, \\
& \frac{d z}{d t}=\delta z-\frac{p_{2}(1-\beta) \delta s}{2 \alpha}+\frac{a r \beta x^{2} \mu_{1}}{2 \alpha(L-a s)^{2}} .
\end{aligned}
$$

Now we try to find the non-negative equilibria of the system of differential equations (2.1). Clearly we see that there may be two equilibria of the system (2.1). One is $P_{1}\left(0,0, p_{1} q E /(\delta+r-q E), 0\right), \quad$ and other is $P_{2}\left(x^{*}, z^{*}, \mu_{1}^{*}, s^{*}\right)$, where $P_{2}$ is given by as follows:

$$
\begin{aligned}
x^{*} & =\frac{r-q E}{r}\left(L-a s^{*}\right), \\
z^{*} & =0, \\
\mu_{1}^{*} & =\frac{p_{1} q E}{\delta+r-q E}, \\
s^{*} & =\frac{a p_{1} q E \beta(r-q E)^{2}}{p_{2} r \delta(1-\beta)(\delta+r-q E)} .
\end{aligned}
$$

For both economical and biological context the necessary and sufficient condition for the existance of the above equilibrium is given by $E<r / q$. If $E>r / q$ then the density of the population $x$ will be negative and so there will be no population in the system. This case may be occured due to the over harvesing than the population intrinsic growth rate and in this case the system will be collapsed. So to remain the system alive we must take the harvesting effort, $E$ less than $r / q$.

\subsection{Stability Analysis of the System.}

In this subsection we now describe the local stability and bifurcation of our system around two equilibria $P_{1}$ and $P_{2}$ in the following theorems.

Theorem 1. The system (2.1) is unstable around the equilibrium $P_{1}$.

Proof. The characteristic equation to the system(2.1) at its equilibrium $P_{1}$ is given by:

$$
(\lambda+r+q E)(\lambda-(\delta-r+q E))\left(\lambda^{2}-\lambda \delta+\frac{p_{2}(1-\beta) \delta}{2 \alpha}\right)=0
$$

i.e. the eigenvalues are given by: $\lambda_{1}=-(r+q E), \lambda_{2}=\delta-r+q E \quad$ and $\lambda_{3,4}=\frac{1}{2}\left(\delta \pm \sqrt{\delta^{2}-2 p_{2}(1-\beta) \delta / \alpha}\right)$. Since the real part of $\lambda_{3,4}$ are positive hence we may conclude that the system (2.1) is unstable at the equilibrium $P_{1}$.

Next we shall study the local stability criterion of the system (2.1) at the equilibrium $P_{2}$. For this we now try to find the characteristic roots of (2.1) at $P_{2}$. After some simple manipulation we get the characteristic equation of (2.1) at $P_{2}$ as

$$
\lambda^{4}+a_{1} \lambda^{3}+a_{2} \lambda^{2}+a_{3} \lambda+a_{4}=0
$$

where

$$
\begin{gathered}
a_{1}=1+q E-\frac{q E}{r}-r-2 \delta, \\
a_{2}=A+2 q E+\frac{2 q E \delta}{r}+r \delta+\delta^{2}-\frac{q^{2} E^{2}}{r}-r-2 \delta-q E \delta, \\
a_{3}=A q E+r \delta+\delta^{2}+\frac{q^{2} E^{2} \delta}{r}+\frac{A(r-q E)}{r}-A r-A \delta-2 q E \delta-\frac{q E \delta^{2}}{r},
\end{gathered}
$$




$$
\begin{aligned}
& a_{4}=A(q E-r)+\frac{A(q E-\delta)(r-q E)}{r}+\frac{a^{2} \beta \mu_{1}^{*}(r-q E)^{4}}{\alpha r\left(L-a s^{*}\right)} \\
& +\frac{a^{2} \beta x^{*} \mu_{1}^{*}(r-q E)^{2}(2 q E-r-\delta)}{\alpha\left(L-a s^{*}\right)^{2}}-\frac{a^{2} \beta x^{*} \mu_{1}^{*}(r-q E)^{3}}{\alpha r\left(L-a s^{*}\right)^{2}}, \\
& A=\frac{\delta(1-\beta) p_{2}}{2 \alpha \beta}-\frac{a^{2} \beta(r-q E)^{2} \mu_{1}^{*}}{\alpha r\left(L-a s^{*}\right)} .
\end{aligned}
$$

Now let us calculate the value of $a_{1} a_{2}-a_{3}$.

We have, $a_{1} a_{2}-a_{3}=-A \delta+a_{21} a_{1}+a_{31}$ where $a_{21}=a_{2}-A$ and $a_{31}=a_{3}-A\left(a_{1}+\delta\right)$.

Thus for $a=a^{*}$ where $a^{*}$ is the positive value of $a$ of the equation $A=\left(a_{1} a_{21}+a_{31}\right) / \delta$ we have, $a_{1} a_{2}-a_{3}=0$.

Now we are in a position to state the following two theorems related to the local stability and Hopf bifurcation to the system (2.1) which can be easily proved by using Routh-Hurwitz criterion.

Theorem 2. The system (2.1) is locally asymptotically stable at $P_{2}$ if $a_{1} a_{2}-a_{3}>0$ and $a_{3}\left(a_{1} a_{2}-a_{3}\right)-a_{4} a_{1}^{2}>0 \quad$ hold provided $a_{i}>0,(i=1,2,3,4)$.

Theorem 3. The system (2.1) undergoes through a Hopf bifurcation at the equilibrium $P_{2}$ for $a=a^{*}$.

\subsection{Influence of S ome Impor tant Parameters to the}

\section{Equilibrium $P_{2}$}

We now intend to see the influence of some important parameters namely $a, E, r$ and $\delta$ at the equilibrium level of the system(2.1).

$$
\begin{aligned}
& \text { We see that } \frac{\partial x^{*}}{\partial a}=-\frac{(r-q E) s^{*}}{r}(<0) \text { and } \\
& \frac{\partial s^{*}}{\partial a}=\frac{p_{1} q E(r-q E)^{2}}{p_{2} r \delta(\delta+r-q E)}(>0) .
\end{aligned}
$$

Thus at the equilibrium $P_{2}$ the value of $x$ decreases(increases) as the parameter $a$ increases(decreases) where as the value of $s$ increases(decreases) as $a$ increases(decreases) although $a$ has no effect on the shadow price $\mu_{1}$.

Also,

$$
\begin{gathered}
\frac{\partial x^{*}}{\partial E}=-\frac{q(L-a s)}{r}(<0), \\
\frac{\partial \mu_{1}^{*}}{\partial E}=\frac{p_{1} q(r+\delta)}{(r+\delta-q E)^{2}}(>0)
\end{gathered}
$$

and

$$
\begin{aligned}
& \frac{\partial s^{*}}{\partial E}=\frac{a q p_{1}(r-q E)\{q E(r-q E)+(r-3 q E)(r+\delta-q E)\}}{r \delta p_{2}(r+\delta-q E)^{2}} . \\
& \text { It is obvious that } \frac{\partial s^{*}}{\partial E}(>0) \text { for } E<E_{1} \text { and } \\
& \frac{\partial s^{*}}{\partial E}(<0) \text { for } E \in\left(E_{1}, r / q\right), \text { provided } E_{1}<r / q \\
& \text { where } E_{1}=\frac{3(r+\delta)+\sqrt{(r+\delta)(r+9 \delta)}}{4 q} .
\end{aligned}
$$

Thus it is concluded that at $P_{2}, x$ decreases(increases) and $\mu_{1}^{*}$ increases(decreases) as $E$ increases(decreases) where as in $E \in\left(0, E_{1}\right), s$ increases(decreases) and for $E \in\left(E_{1}, r / q\right), \quad s$ decreases(increases) as $E$ increases(decreases).

Next,

$$
\frac{\partial x^{*}}{\partial r}=\frac{q E(L-a s)}{r^{2}},
$$

$$
\begin{aligned}
\frac{\partial \mu_{1}^{*}}{\partial r} & =-\frac{p_{1} q E}{(r+\delta-q E)^{2}}(<0), \\
\frac{\partial s^{*}}{\partial r} & =\frac{a q p_{1} E(r-q E)\{\delta(r+q E)+q E(r-q E)\}}{p_{2} \delta r^{2}(r+\delta-q E)^{2}}(>0) .
\end{aligned}
$$

Thus we conclude, at $P_{2}, \quad x$ and $s$ both increases(decreases) where as the shadow price $\mu_{1}$ decreases(increases) as the intrinsic growth rate $r$ increases(decreases).

Ultimately, $\quad \frac{\partial \mu_{1}^{*}}{\partial \delta}=-\frac{p_{1} q E}{(r+\delta-q E)^{2}}(<0) \quad$ and $\frac{\partial s^{*}}{\partial \delta}=-\frac{a q p_{1} E(r-q E)^{2}(r+2 \delta-q E)}{p_{2} r \delta^{2}(r+\delta-q E)^{2}}(<0)$.

Thus at $P_{2}$ both $\mu_{1}$ and $s$ decreases(increases) as the parameter $\delta$ increases(decreases) although $\delta$ has no influence on the population density $x$.

\section{Analysis of the System for}

\section{Cobb-Douglous Catching Function}

$$
h=q E^{2}
$$

Again using the optimality condition $\partial H / \partial z=0$ we get the reduced form of system of differential equations (1.6) as follows: 


$$
\begin{aligned}
& \frac{d x}{d t}=r x\left(1-\frac{x}{L-a s}\right)-q E^{2}, \\
& \frac{d s}{d t}=\beta z, \\
& \frac{d \mu_{1}}{d t}=\left(\delta-r+\frac{2 r x}{L-a s}\right) \mu_{1}, \\
& \frac{d z}{d t}=\delta z-\frac{p_{2}(1-\beta) \delta s}{2 \alpha}+\frac{\operatorname{arx}^{2} \mu_{1}}{2 \alpha(L-a s)^{2}} .
\end{aligned}
$$

Next we try to find all possible non-negative equilibria of the above system by making the right hand side of above equation (3.1) equals to zero. Clearly the system (3.1) has three different equilibria and among them the first two are respectively $P_{4}(\bar{x}, 0,0,0)$ and $P_{41}(\hat{x}, 0,0,0)$ where $\bar{x}$ and $\hat{x}$ are the roots of the equation $r x^{2}-r L x+L q E^{2}=0$. The rest equilibrium is given by $P_{5}\left(x^{\prime}, 0, \mu_{1}^{\prime}, s^{\prime}\right), \quad \quad \quad \quad \quad x^{\prime}=\frac{2 q E^{2}}{r+\delta}$, $\mu_{1}^{\prime}=\frac{4 p_{2}(1-\beta) r}{a^{2}(r-\delta)^{2}}\left[L-\frac{4 r q E^{2}}{r^{2}-\delta^{2}}\right]$, $s^{\prime}=\frac{1}{a}\left[L-\frac{4 r q E^{2}}{r^{2}-\delta^{2}}\right]$. Now both equilibria $P_{4}$ and $P_{41}$ are either feasible or imaginary depending on the nature of $x^{\prime}$. If $r L-4 q E \geq 0$ then both equilibria $P_{4}$ and $P_{41}$ are feasible otherwise they are imaginary. Now both the equilibria $P_{4}$ and $P_{41}$ has only biological impact since the population are able to survive. On the otherhand these equilibria have no economic meaning due to the aqualture and only economic profit may be gained by the harvesting.

\subsection{Stability Analysis}

In this section we shall discuss the stability of the system (3.1) at different equilibria. In the following theorem we study the local stability of the system around $P_{4}$ and $P_{41}$. Next we study the same around $P_{5}$.

Theorem 4. The system (3.1) is unstable around both the equilibria $P_{4}$ and $P_{41}$.

Proof. The characteristic equation to the system (3.1) at the equilibrium $P_{4}$ is given by:

$$
\lambda^{4}+b_{1} \lambda^{3}+b_{2} \lambda^{2}+b_{3} \lambda+b_{4}=0
$$

where,

$$
\begin{gathered}
b_{1}=-2 \delta \\
b_{2}=-r^{2}\left(1-\frac{2 \bar{x}}{L}\right)^{2}+r\left(1-\frac{2 \bar{x}}{L}\right) \delta+\delta^{2}+\frac{\delta p_{2}(1-\beta)}{2 \alpha}, \\
b_{3}=r^{2}\left(1-\frac{2 \bar{x}}{L}\right)^{2} \delta-r\left(1-\frac{2 \bar{x}}{L}\right) \delta^{2}-\frac{\delta^{2} p_{2}(1-\beta)}{2 \alpha}
\end{gathered}
$$

$$
b_{4}=\frac{r}{2 \alpha}\left(1-\frac{\bar{x}}{L}\right) \delta p_{2}(1-\beta)\left(\delta-r\left(1-\frac{2 \bar{x}}{L}\right)\right) .
$$

Since here $b_{1}$ is negative then using Routh-Hurwitz criterion for stability we may say that at least one eigenvalue of the system (3.1) at the equilibrium $P_{4}$ is positive. Hence the system is unstable around $P_{4}$. Similarly replacing $\bar{x}$ by $\hat{x}$ in the characteristing equation (3.2) we can obtain the characteristic equation of (3.1) around $P_{41}$. The coefficient of third degree term of that characteristic equation also will be $-2 \delta(<0)$. Thus with the help of previous argument we can conclude that the equilibrium $P_{41}$ is also unstable. Hence the theorem.

In the next theorem we will study the stability of the other equilibrium $P_{5}$ of the system (3.1)

Theorem 5. The system (3.1) is unstable around the equilibrium $P_{5}$.

Proof. The characteristic equation of the system (3.1) at the equilibrium $P_{5}$ can be written as follows:

$$
\lambda^{4}+c_{1} \lambda^{3}+c_{2} \lambda^{2}+c_{3} \lambda+c_{4}=0
$$

where

$$
\begin{gathered}
c_{1}=-2 \delta, \\
c_{2}=\delta^{2}+\frac{\delta p_{2}(1-\beta)}{2 \alpha}-\frac{a^{2}(r-\delta)^{3} \mu_{1}^{\prime}}{8 r^{2} \alpha x^{\prime}}, \\
c_{3}=-\frac{\delta p_{2}(1-\beta)}{2 \alpha}+\frac{a^{2}(r-\delta)^{3} \mu_{1}^{\prime}}{8 r^{2} \alpha x^{\prime}}, \\
c_{4}=\frac{a^{2}(r-\delta)^{5} \mu_{1}^{\prime}}{32 r^{2} \alpha x^{\prime}}+\frac{a^{2}(r-\delta)^{4} \delta \mu_{1}^{\prime}}{16 r^{2} \alpha x^{\prime}} .
\end{gathered}
$$

The characteristic equation (3.3) shows us that at least one eigen value of this characteristic equation is positive (using Routh Hurwitz critiaria for a polynomial equation of degree four). Hence the system (3.1) is unstable around its equilibrium $P_{5}$. Hence the theorem.

Note. It is evident that if the catching would be of the form of Cobb-Douglous production function then always ststem would be unstable around its all possible feasible equilibria.

$$
\text { 4. Analysis for } h(t)=\frac{q E x(t)}{m E+n x(t)}
$$

Here we take the catching function of Michaelis-Menten type i.e. of the form $h=q E x /(m E+n x)$ where $q$ is the catchibility coefficient and $m, n$ are the Michaelis-Menten constants. So from (1.6) we have the four first order differential equations which can be deduced for harvesting by Michaelis-Menten type by using the optimal conditions are as follows: 


$$
\begin{aligned}
& \frac{d x}{d t}=r x\left(1-\frac{x}{L-a s}\right)-\frac{q E x}{m E+n x} \\
& \frac{d s}{d t}=\beta z \\
& \frac{d \mu_{1}}{d t}=\mu_{1}\left(\delta-r+\frac{2 r x}{L-a s}\right)-\frac{\left(p_{1}-\mu_{1}\right) m q E x}{(m E+n x)^{2}}, \\
& \frac{d z}{d t}=\delta z-\frac{p_{2}(1-\beta) \delta s}{2 \alpha}+\frac{a r x^{2} \mu_{1}}{2 \alpha(L-a s)^{2}} .
\end{aligned}
$$

Next we try to find the possible non-negative equilibria of the system (4.1) by making right hand side of system (4.1) equal to zero. We are interested about the interior equilibrium of the system (4.1) although we always have $z=0$ at the equilibrium. So let us denote the non zero value of $x, \mu_{1}, s$ at the interior equilibrium of the system (4.1). Let $P_{3}\left(x_{1}, 0, \mu_{11}, s_{1}\right)$ where $x_{1}$ is the positive root of the equation

$$
A_{1} x^{4}+A_{2} x^{3}+A_{3} x^{2}+A_{4} x+A_{5}=0
$$

where,

$$
\begin{gathered}
A_{1}=n^{4} r \delta p_{2}(r+\delta) \\
A_{2}=m n^{2} q E r p_{2} \delta+4 m n^{3}(r+\delta) E \delta p_{2}-2 r q E n^{3} \delta p_{2} \\
A_{3}=6 E^{2} m^{2} n^{2} r \delta(r+\delta) p_{2}-6 E^{2} m n^{2} q r \delta p_{2} \\
+2 E^{2} m^{2} n q r \delta p_{2}-a E^{2} m n^{2} q r^{2} p_{1} \\
A_{4}=4 E^{3} m^{3} n r \delta(r+\delta) p_{2}-6 E^{3} m^{2} n r q \delta p_{2} \\
+E^{3} m^{3} q r \delta p_{2}+2 a E^{3} m n q r(m r-q) p_{1}
\end{gathered}
$$

and

$$
\begin{aligned}
A_{5}= & E^{4} m^{4} r \delta(r+\delta) p_{2}-2 E^{4} m^{3} r q \delta p_{2} \\
& -a E^{4} m q(m r-q)^{2} p_{1} .
\end{aligned}
$$

Clearly a sufficient condition that (4.2) has only one positive root is $A_{2} A_{5}<0$ and $A_{3} A_{4}>0$ hold simultaneously. Also $\mu_{11}=\frac{p_{2} \delta}{a r}\left[\frac{r\left(m E+n x_{1}\right)}{(m r-q) E+r n x_{1}}\right]^{2}$ and $s_{1}=\frac{1}{a}\left[L-\frac{r x_{1}\left(m E+n x_{1}\right)}{(m r-q) E+r n x_{1}}\right]$.

Thus the equilibrium $P_{3}\left(x_{1}, 0, \mu_{11}, s_{1}\right)$ is always nonnegative if (4.2) has one positive root and $L\left\{(m r-q) E+r n x_{1}\right\}>\left\{r x_{1}\left(m E+n x_{1}\right)\right\}$.

\subsection{Stability Analysis}

Here we now discuss the criteria for local stability and examine if there will be any bifurcation at the equilibrium $P_{3}\left(x_{1}, 0, \mu_{11}, s_{1}\right)$ of the system (4.1). The characteristic equation to the system (4.1) around its equilibrium $P_{3}\left(x_{1}, 0, \mu_{11}, s_{1}\right)$ is given by:

$$
\lambda^{4}+B_{1} \lambda^{3}+B_{2} \lambda^{2}+B_{3} \lambda+B_{4}=0
$$

where

$$
\begin{aligned}
& B_{1}=q E B_{6}-2 \delta-q E x_{1}(m+n) B_{6}^{2}, \\
& B_{2}= \delta+\delta^{2}-r^{2}+\delta p_{2} /(2 \alpha)+2 r x_{1}(r-\delta) B_{5} \\
&- 4 r^{2} x_{1}^{2} B_{5}^{2}+m n q^{2} E^{2} x_{1}^{2} B_{6}^{4}-m q^{2} E^{2} x_{1} B_{6}^{3}+ \\
& q E x_{1}(m r+m \delta+2 n \delta-n r) B_{6}^{2}-2 q E r x_{1}^{2}(m-n) B_{5} B_{6}^{2} \\
&- 2 q E r x_{1} B_{5} B_{6}+q E(r-2 \delta) B_{6}, \\
& B_{3}= r \delta(r-\delta)-4 r^{2} x_{1} \delta B_{5}+4 r^{2} x_{1}^{2} \delta B_{5}^{2} \\
&-m n q^{2} E^{2} x_{1}^{2} \delta B_{6}^{4}+m q^{2} E^{2} x_{1} \delta B_{6}^{3} \\
&-m q E r x_{1} \delta(m-n) B_{6}^{2}+2 q E r x_{1}^{2} \delta(m-n) B_{5} B_{6}^{2} \\
&-q E \delta(r-\delta) B_{6}+2 q E r x_{1} \delta B_{5} B_{6}+2 r x_{1} \delta^{2} B_{5} \\
&-n q E x_{1} \delta^{2} B_{6}^{2}+B^{\prime}\left(\delta-q E B_{6}+q E x_{1}(m+n) B_{6}^{2}\right), \\
& B_{4}= m q^{2} E^{2} x_{1} B^{\prime} B_{6}^{3}\left(1-n x_{1} B_{6}\right)+n q E(r-\delta) x_{1} B^{\prime} B_{6}^{2} \\
&+ 2(m-n) q E r x_{1}^{2} B^{\prime} B_{5} B_{6}^{2}-q E r B^{\prime} B_{6}\left(1-2 x_{1} B_{5}\right)+ \\
& r^{2} B^{\prime}-m q E r x_{1} B^{\prime} B_{6}^{2}+r x_{1} B^{\prime} B_{5}(\delta-2 r)+q E \delta B^{\prime} B_{6} \\
&+ a^{2} r^{2} x_{1}^{3} \mu_{11} B_{5}^{4}\left(2 r-3 r x_{1} B_{5}-\delta\right) / \alpha- \\
& a^{2} q E r^{2} x_{1}^{3} \mu_{11} B_{5}^{4} B_{6}\left(1-n x_{1} B_{6}\right) / \alpha+m q E a^{2} r^{2} x_{1}^{4} B_{5}^{4} B_{6}^{2} \\
&\left(2 n x_{1}\left(p_{1}-\mu_{11}\right) B_{6}-\left(p_{1}+\mu_{11}\right)\right) /(2 \alpha), \\
& B_{5}=1 /\left(L-a s_{1}\right), B_{6}=1 /\left(m E+n x_{1}\right) \text { and } \\
& B^{\prime}=\left(-\delta p_{2} /(2 \alpha)+a^{2} r x_{1}^{2} \mu_{11} B_{5}^{3} / \alpha\right) . \\
& \\
& \\
& \\
&
\end{aligned}
$$

Next we use the well known Routh-Hurwitz criteria to find the nature of the stability of the system around its equilibrium $P_{3}$. For this at first we define the following quantities:

$$
\begin{gathered}
J_{1}=B_{1}, J_{2}=B_{1} B_{2}-B_{3}, \\
J_{3}=B_{1} B_{2} B_{3}-B_{3}^{2}-B_{1}^{2} B_{4} .
\end{gathered}
$$

Now we state the following theorem in connection with the local stability criteria to the system (4.1) around its equilibrium point $P_{3}$ which can be proved by using the well known Routh-Hurwitz criterion for the local stability.

Theorem 6. If all of $B_{1}, B_{3}, B_{4}, J_{2}$ and $J_{3}$ are positive then the system (4.1) is locally asymptotically stable around $P_{3}$ where as if at least one of them is negative then the system (4.1) is unstable around $P_{3}$.

\section{Numerical Simulation}



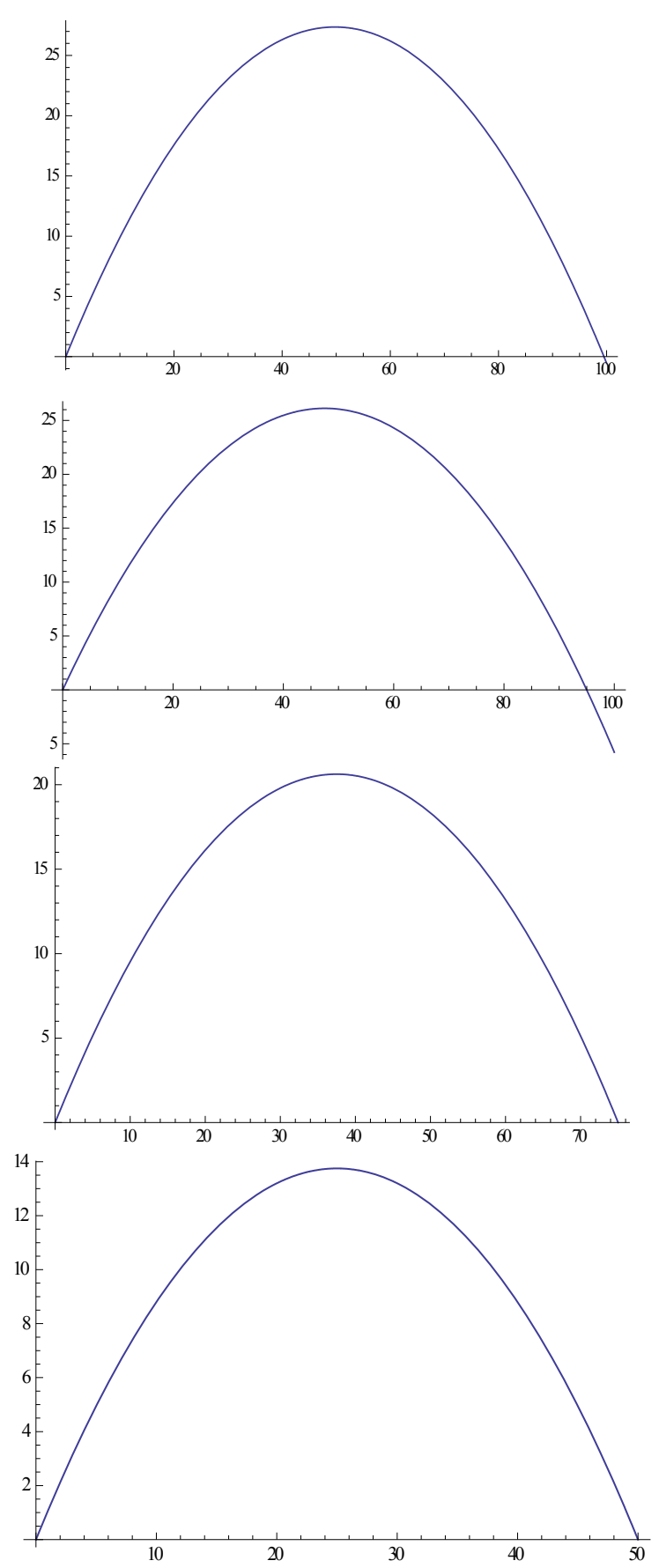

Figure 2. Graph of the population biomass for $a=0.1,1,5,10$

respectively where $x$ is taken along the horizontal axis and the increment of $x$ is taken along the verticle axis

In this section we not only verify our analytical results through computer simulation but also we present some realistic phenomena for different parameter values.

First we check the population biomass level for different value of the parameter $a$ which is associated with the eco-tourism region $s$. For the simulation purpose we take $r=1.1, L=100$ and four different values of $a$ namely $0.1,1,5$ and 10 with fixed value of $s$ as 5 units. In figure 2 , we present the phenomena and see that higher values of $a$ makes to decrease population biomass quickly.
Next we draw the graph for population growth curve and different catching function for different effort. Here for simulation we take, $r=3.1, L=100, a=5, q=0.2, m=0.1, n=0.01$. Also we take a fixed value of $s=5$. In figure 3, 4 and 5 we represent these phenomena for different effort and different harvesting rate respectively.

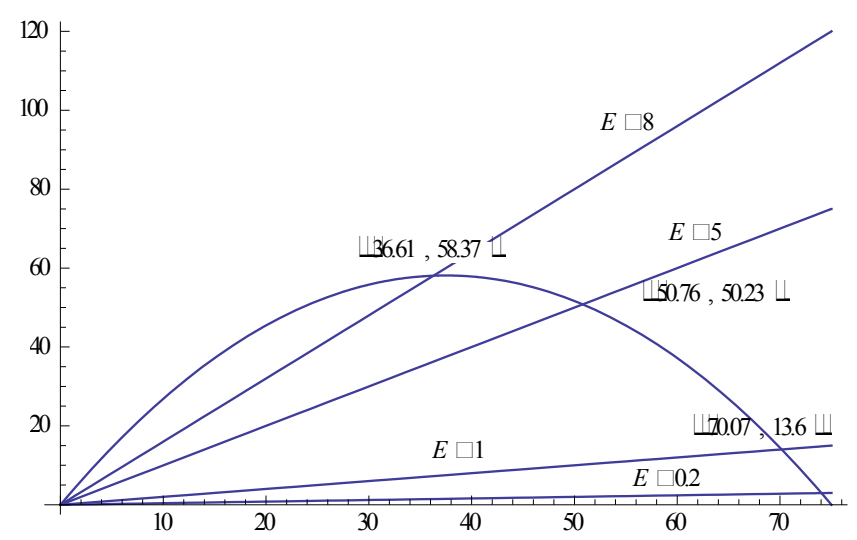

Figure 3. Graph for catching according to Schaefer catching function where $x$ is taken along the horizontal axis and $d x / d t$ is taken along the verticle axis

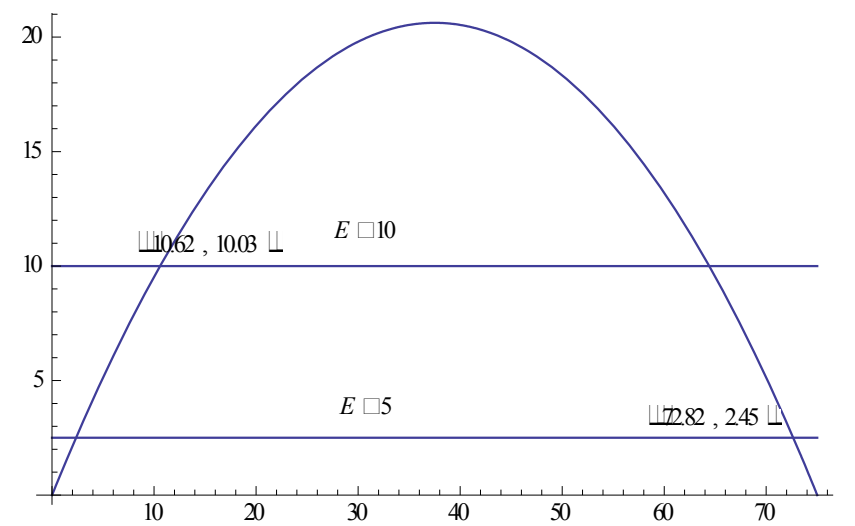

Figure 4. Graph for catching according to Cobb-Douglous catching function where $x$ is taken along the horizontal axis and $d x / d t$ is taken along the verticle axis

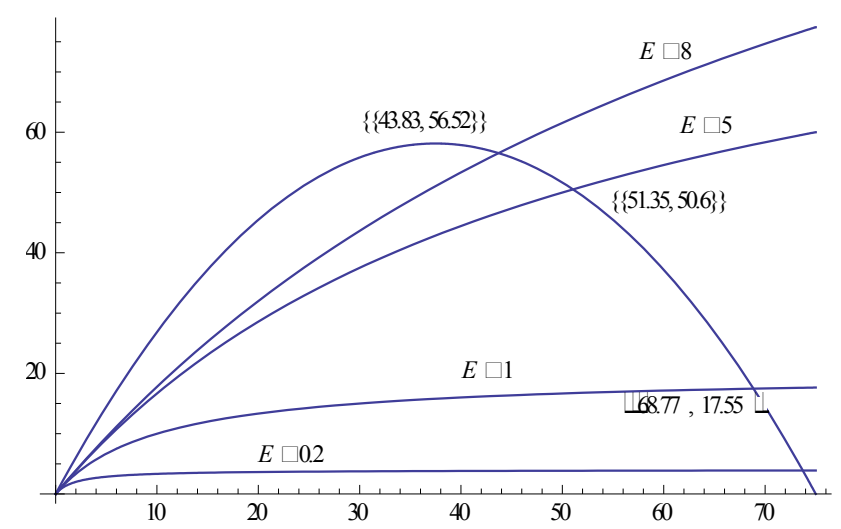

Figure 5. Graph for catching according to Michaelis-Menten catching function where $x$ is taken along the horizontal axis and $d x / d t$ is taken along the verticle axis

The above three figures indicate the biomass of the 
population at the equilibrium level when we use different rate of harvesting. It is seen that the worst population density would be for catching according to Cobb-Douglus production function. Again catching according to the Michaelis-Menten rule gives the best result because here we can control both population biomass and total harvesting populations together since there are two extra control parameter namely $m, n$ in this type of harvesting.

\section{Conclusions}

For a fishery manager the management objective is to maximize the rent of the fishery using certain management tools. On the otherhand long term resource conservation is also very much essential for our future generation as well. Under this circumstences marine reserve (where fishing is strictly prohibited) may be considered as an essential tool for this management purposes. We can develop ecotourism to overcome dilemma between the need for long term resource conservation and the immid iate necessity to provide jobs and income to the local population. In the present work we consider one portion of the available region to be used for ecotourism purpose (where fishing is not permitted) and the rest part to be used for fishery management. Here the region which is considered for ecotourism purpose is not a constant region. In fact this region is proportional to a particular area $S$ whose cost to make it suitable for tourism purpose is known. A lso the investment cost to this zone is divided into two parts: one part is applied to make the zone usable for tourism and the rest part is used as secondary cost to make the zone more attractive. Obviously this secondary cost would be proportional to the profit earn from the tourism purpose. Our main aim is to maximize the total profit from both the fishery and ecotourism. This type of simultaneous fishery and ecotourism policy would be very helpful to marine areas particularly nearby of any coastal areas or bank of the see. The idea of simultaneous fishing and ecotourism help to maintain equaility in the marine population. Thus to describe marine ecology our analys is would be very handful.

Here we consider three types of catch rate functions, the Schaefer catching function $(h=q E x)$, the Cobb-Douglous production function $\left(h=q E^{2}\right)$ and the Michaelis-Menten type catching which is of the form $h=q E x /(m E+n x)$. We analyze the stability of the systems at all feasible optimal equilibria. Among all the catching function the Michaelis-Menten type catching i.e. $h=q E x /(m E+n x)$ is most suitable catching function because in this case neither population nor harvesting effort go to infinity. Again it is evident from our throughout analys is that at the optimu m equilibriu m level the cost for the eco-tourism is zero.

When we harvest according to the Schaefer catching function $h=q E x$, the system (2.1) gives two optimal equilibria, one is the population free equilibrium $P_{1}$ and another is $P_{2}$. If the growth rate of the population is less than the harvesting, then the system goes to a population free position and in this case system goes to the equilibrium $P_{1}$ which is a saddle point. A long $P_{1}$ all the trajectories except the trajectory along $x$ axis repel from $P_{1}$. The trajectory along $x$ axis attracted at $P_{1}$ due to the high harvesting rate as well as lower growth rate. Again the optimal equilibrium $P_{2}$ is conditionally locally asymptotically stable and for a critical value of $a$ the system (2.1) undergoes through a Hopf bifurcation.

We see that when we use the Cobb-Douglous production function as the catching function then the reduced systemhas three possible non negative equilibria depending upon some parametric conditions and the system is unstable in nature around its all of those three possible equilibria. This is possibly due to the rate of the harvesting as the quadratic function of the fishing effort. Since harvesting is occurring at a rate of $q E^{2}$, the population decreases always and this causes the increasing of the space for eco-tourism. Thus this type of harvesting never stabilize the population density and the eco-tourism region.

Next when we use Michaelis-Menten type harvesting then the harvesting function is not only proportional to the harvesting effort and the stock of the population biomass but also it is inversely proportional to the joint effect of the harvesting effort and population biomass. In this type of Harvesting we also obtain more than one equilibria but we are interested to examine the system nature around the interior one and so we omit the others from our discussion. We find the local asymptotic stability criteria of the reduced optimal system around that interior equilibrium $P_{3}$ and see that it is conditionally stable there. Other functional form embodies like either $h=q E x$ or $h=q E^{2}$ has the following defects: (i) assumes random search for fish, (ii) assumes equallikelihood of be ing captured for every fish,(iii) there is unbounded linear increase in $h$ with respect to $E$ for a fixed $x$, (iv) there is unbounded linear increase in $h$ with respect to $x$ for a fixed $E$ etc. But those unrealistic features can be largely removed by adapting the alternative functional form of harvesting type of harvesting $h=q E x /(m E+n x)$. Hence this type of functional form is the best for both biological and economical context.

In traditional way we use to harvest a population species to get some benefit from it. But over harvesting and unconsciousness harvesting causes the imbalance of natural resource of life because it may be the cause for the extinction of one or more species. Therefore to conserve the population for the future generation we may introduce some marine reserve where fishery may not be permitted but reserved region may be used for the purpose of the ecotourism. This ecotouris m is economically profitable for the local people as wll as the government. In this way we see that eco-tourism is 
not only economically beneficial but also biologically strongly essential and this would make a balance of the population level in marine ecology.

\section{ACKNOWLEDGEMENTS}

Research of Soovoojeet Jana is financially supported by University Grants Commission, Government of India (F. 11-2/2002 (SA-1) dated 19 August, 2011). [3]

\section{REFERENCES}

[1] J. M. Conrrad, The bioeconomics of marine sanctuaries, Journal of Bioeconomics, 1, 206-217, (1999).

[2] T. Luke, C.W. Clark, M. Manget and G. R. Munro, Implementing the precautionary principles in fisheries management through marine reserves, Ecological Applications, 8(1), 1998, 72-78.

[3] T. K. Kar and H. Matsuda, A bioeconomic model of a single species fishery with a marine reserve, Journal of Enviornmental Management, 86, 2008, 171-180.

[4] K. Hartmann, L. Bode and P. Armsworth, The economic optimality of learning from marine protected areas, ANZIAM, 48, 2007, 307-329.
[5] I. Rodwell and E. Barbier, A model of tropical marine reserve-fishing linkages, Natural Resource Modelling, 15(4), 2002 453-486.

[6] T. K. Kar and K. Chakraborty, Marine reserves and its consequences as a fisheries management tool, World Journal of Modelling and Simulation, 5(2), 2009, 83-95.

[7] M. L. Vilchez, F. Velasco and I. Herrero, An optimal control problem with Hopf bifurcations: an application to the striped venus fishery in the Gulf of Cadiz, Fisheries Research, 67, 2004, 295-306.

[8] K. R. Stollery, Monopsony processing in an open access fishery, Marine Resource Economics, 3(4), 1986, 331-351.

[9] G. Feichtinger and G. Sorger, Optimal oscillation in control models: how can constant demand lead to cyclical production?, Oper. Res. Let. 5, 1986 270-281.

[10] M. B. Schaefer, Some consideration of population dynamics and economics in relation to the management of marine fisheries, J. Fish. Res. Board Can., 14, (1957) 669-681.

[11] C. W. Clark, Mathematical Bioeconomics: The optimal management of renewable resources. New York: Wiley Series (1990).

[12] M. Kot, Elements of Mathematical Ecology, Cambridge University Press (2001). [4] 